Article - Human and Animal Health

\title{
Evaluation of Toxocara canis Glycosylated TES Produced in Pichia pastoris for Immunodiagnosis of Human Toxocariasis
}

Lucas Moreira dos Santos ${ }^{1^{*}}$

https://orcid.org/0000-0002-2402-8580

Michele Pepe Cerqueira ${ }^{1}$

https://orcid.org/0000-0003-2138-0703

Giana Carla Gaboardi ${ }^{1}$

https://orcid.org/0000-0001-5153-5468

Carolina Georg Magalhães ${ }^{1}$

https://orcid.org/0000-0001-7099-060X

Rafael Amaral Donassolo'

https://orcid.org/0000-0002-9919-6845

Rafael Rodrigues Rodrigues ${ }^{1}$

https://orcid.org/0000-0002-1453-2898

\section{Emili Griep ${ }^{1}$}

https://orcid.org/0000-0002-6962-7640
Marcos Roberto Ferreira ${ }^{1}$

https://orcid.org/0000-0002-6658-7196

Guita Rubinsky Elefant ${ }^{2}$

https://orcid.org/0000-0003-4289-2169

Luciana Farias da Costa Avila ${ }^{3}$

https://orcid.org/0000-0003-2171-7046

Carlos James Scaini ${ }^{3}$

https://orcid.org/0000-0001-7998-0256

Ângela Nunes Moreira ${ }^{1}$

https://orcid.org/0000-0001-5347-8559

Fabricio Rochedo Conceição ${ }^{1}$

https://orcid.org/0000-0002-1072-4374

1 Universidade Federal de Pelotas, Departamento de Biotecnologia, Capão do Leão, Rio Grande do Sul, Brazil; 2Universidade de São Paulo, Instituto de Medicina Tropical de São Paulo, São Paulo, São Paulo, Brazil; ' $U n i v e r s i d a d e$ Federal do Rio Grande, Faculdade de Medicina, Rio Grande, Rio Grande do Sul, Brazil.

Received: 2019.03.12; Accepted: 2020.06.01.

*Correspondence: lucass1@hotmail.com; Tel.: +55-53-984182790 (L.M.S.)

\section{HIGHLIGHTS}

- Pichia pastoris rTES evaluated as an alternative tool for toxocariasis diagnosis.

- No difference between sensitivities of glycosylated and partial glycosylated rTES.

- High-throughput expression and in vivo purification of rTES in Pichia pastoris.

Abstract: Recombinant proteins are a suggested alternative for the diagnosis of toxocariasis. The current Escherichia coli recombinant protein overexpression system usually produces insoluble products. As an alternative, yeast such as Pichia pastoris have secretory mechanisms, which could diminish the cost and time for production. This study aimed to produce recombinant proteins in Pichia pastoris and verify their sensibility and specificity in an indirect ELISA assay. Two sequences (rTES-30 and rTES-120) of Toxocara canis excretory-secretory antigens were cloned in a pPICZaB vector and expressed in $P$. pastoris $\mathrm{KM} 71 \mathrm{H}$. Sera samples collected from human adults infected by Toxocara spp. were tested by indirect ELISA using rTES-30 and rTES-120 as antigens. Recombinant proteins were detected at 72 hours after induction, in the 
supernatant, as pure bands between $60 \sim 70 \mathrm{kDa}$ with hyperglycosylation. Regarding diagnosis potential, recombinant antigens had high specificity (95.6\%); however, sensitivity was $55.6 \%$ for rTES-30 and $68.9 \%$ for rTES-120. Further deglycosylation of the $P$. pastoris antigens did not seem to affect ELISA performance ( $p>0.05$ ). The low sensitivity in the serodiagnosis diminished any advantage that $P$. pastoris expression could have. Therefore, we do not recommend $P$. pastoris recombinant TES production as an alternative for the diagnosis of toxocariasis.

Keywords: diagnosis; ELISA; recombinant protein; Pichia pastoris; glycosilation; toxocariasis.

\section{INTRODUCTION}

Toxocariasis is a neglected zoonosis with a worldwide distribution and is transmitted to animals and humans by infectious eggs from Toxocara canis and Toxocara cati. Infected patients may develop clinical symptoms according to larvae migration, obscuring or even invaliding clinical diagnosis. The traditional diagnostic method for this disease relies on Enzyme Linked Immune Sorbent Assay (ELISA) and Western Blotting [1]. While this laboratory method is viable and cost-effective, the production of $T$. canis antigens is a major disadvantage that restricts its accessibility because the antigen production is arduous, demands highly specialized laboratory equipment and personnel, and requires a minimum of 60 days [2].

As an alternative, recombinant proteins of the secretory and excretory families (TES) have been proposed. Three recombinant proteins (rTES-26, rTES-32, and rTES-120) were produced in Escherichia coli and showed high sensibility and specificity, essential requirements for the diagnosis of toxocariasis in humans [3]. However, E. coli express TES proteins in the cytoplasm and may therefore generate insoluble products, impeding purification treatments and increasing the associated production cost [4].

Expression in Pichia pastoris is an interesting alternative to recombinant TES produced in E. coli. One advantage is the secretory mechanism presented by yeast, which serves as an in vivo purification methodology and reduces the major costs associated with purifying recombinant proteins. Also, E. coli produce TES recombinant proteins in inclusions bodies, which require denaturing conditions and posterior refolding that is evitable in $P$. pastoris [3,5]. Another advantage is that $P$. pastoris can be cultivated in a lowcost medium at a broader $\mathrm{pH}$ range and lower temperatures-essential characteristics for industrial scale [6]. Finally, the last advantage over the $E$. coli system is the possibility of post-translational modifications, especially in the case of native TES, as the proteins are highly glycosylated and could respond to a part of the immune response [7]. Prior to this study, Fong and coauthors presented the recombinant TES-120 expressed in $P$. pastoris with high specificity, suggesting an unexplored potential for TES protein in Toxocara spp. infection diagnosis [8].

For this reason, this study aims to produce recombinant proteins (rTES-30 and rTES-120) in the yeast Pichia pastoris and verify their sensibility and specificity in an indirect ELISA assay.

\section{MATERIAL AND METHODS}

\section{Cloning and Expression of Recombinant Proteins}

To induce the expression of the TES-30 and TES-120 antigens, synthetic genes (Epoch Biolabs, Inc. USA) containing Pichia pastoris preferential codons and restriction enzyme sites were synthesized to direct cloning into the expression vector $P$. pastoris pPICZaB (Invitrogen. USA) according to the sequences deposited in GenBank under accession numbers 4586556 (TES-30) and 1103869 (TES-120).

Synthetic genes were cloned into the pPICZaB vector according to Green and Sambrook [9]. A recombinant clone from each construct was linearized with restriction enzyme Pmel to optimize vector integration by homologous recombination. Pichia pastoris KM71H (MutS) was grown on an orbital shaker at $28{ }^{\circ} \mathrm{C}$ and $120 \mathrm{rpm}$. Competent cells were transformed by electroporation with $10 \mu \mathrm{g}$ of the linearized recombinant vector (pPICZaB-TES-30 and pPICZaB-TES-120) according to Invitrogen (EasySelectTM Pichia Expression Kit, Version G; Invitrogen, USA). The recombinant clones were selected on YPD agar (bacteriological peptone $20 \mathrm{~g} / \mathrm{L}$, yeast extract $10 \mathrm{~g} / \mathrm{L}$, glucose $20 \mathrm{~g} / \mathrm{L}, 15 \mathrm{~g} / \mathrm{L}$ Agar; Synth, Brazil) with Zeocin $500 \mathrm{ug} / \mathrm{mL}$ after incubation for four days at $28^{\circ} \mathrm{C}$.

Transformants were spiked in BMGY broth $(2.0 \%$ peptone, $1.0 \%$ yeast extract, $100 \mathrm{mM}$ potassium phosphate $\mathrm{pH} 6.0,1.34 \%$ yeast nitrogen base without amino acids, $0.4 \mu \mathrm{g} / \mathrm{mL}$ biotin, $1.0 \%$ glycerol; Synth, Brazil) and incubated in the orbital agitator $\left(100 \mathrm{rpm}\right.$ at $\left.28^{\circ} \mathrm{C}\right)$ until they reached $\mathrm{DO}_{600}=4$. At that time, the culture was centrifuged and the pellet was resuspended in BMMY (2\% peptone, $1 \%$ yeast extract, $1.34 \%$ potassium phosphate, $\mathrm{pH} 6.0,100 \mathrm{mM}$ yeast nitrogen base without amino acids, $0.4 \mu \mathrm{g} / \mathrm{mL}$ biotin, $0.5 \%$ 
methanol; Synth, Brazil) containing 0.5\% methanol. For 7 days, $0.5 \%$ methanol was added every 24 hours to the culture of the clones containing pPICZaB-TES-30 and pPICZaB-TES-120. Samples of supernatant and pellet were collected daily. A well-characterized $P$. pastoris clone (pPICZaB-gD) was cultivated as a positive control [10] and native $P$. pastoris $\mathrm{Km} 71 \mathrm{H}$ was cultivated for a negative control; both performed under the same culture conditions described previously. The rTES-30 and rTES-120 proteins were concentrated from the culture supernatant by dialysis (Membra-cell MD34, Viskase, USA) in high osmolarity solution (5M dextrose, Synth, Brazil).

It is important to emphasis that both $P$. pastoris products are the same to a previously published study with E. coli, changing only the expression system, primers details of each product are available on Santos and coauthors study [11].

\section{Deglycosylation}

A fraction of protein volume was submitted to a deglycosylation procedure following the protocol described by Promega (Endoglycosidase H. Promega, USA). Expression levels and digestion were analyzed in the supernatant by sodium dodecyl sulfate-polyacrylamide gel electrophoresis (SDS-PAGE) and Western blotting. The proteins were further quantified with the Pierce BCA kit (Thermo Fisher Scientific, USA).

\section{Sample Collection}

Samples were collected from two distinct blood banks. Blood bank 1 donated samples that were collected from 90 health workers at the Hospital Universitário Dr. Miguel Riet Corrêa Jr in Rio Grande - RS, Brazil and at the Parasitology Laboratory of the Universidade Federal de Pelotas between the years of 2014-2015. Within this collection, 45 health workers were positive for Toxocara spp. infection and 45 were negative. Infection was diagnosed by seropositivity to Toxocara spp. (via native TES ELISA and Western blotting confirmation) and three epidemiological factors: eosinophilia, contact with young dogs/cats, and geophagy. Blood bank 2 donated samples from Laboratório de Soroepidemiologia e Imunobiologia do Instituto de Medicina Tropical de São Paulo (IMT-SP) of Universidade de São Paulo. All sera were found to be negative for Toxocara spp. infection based on epidemiology factors and laboratory assays. Other parasites were diagnosed from clinic data, epidemiology, and laboratory assays, revealing 40 cases with parasite infections (Ascaris lumbricoides, Trichuris trichiura, Ancylostomids, Strongyloides stercoralis, Hymenolepis nana, and Fasciola hepatica) and 40 cases without. All sera were stored at -20C until laboratory assay.

\section{Indirect ELISA}

To verify the antigenicity of the recombinant proteins, we used ELISA. ELISA protocol was optimized prior to the study. Each well of the 96-well flat-bottomed microtiter plate (Nunc Immuno Maxisorp, Thermo Fischer Scientific, USA) was coated with $100 \mathrm{uL}$ of each recombinant antigen at the optimum concentration $(50 \mathrm{ng})$ for each antigen in $0.02 \mathrm{M}$ bicarbonate buffer, $\mathrm{pH}$ 9.6. The plates were then covered and incubated at $4{ }^{\circ} \mathrm{C}$ overnight. The plates were washed with PBS-T, to remove unadsorbed antigen. After a washing step of three washes for 5 min each with PBS-T, each well was blocked with $5 \%$ dry-milk (Nestle, Sweden) PBST solution for $1 \mathrm{~h}$ at $37^{\circ} \mathrm{C}$. The plates were again washed as previously described, followed by the addition of sera samples (100 uL, 1:150 in PBS-T, duplicate wells), and incubated at $37^{\circ} \mathrm{C}$ for $1 \mathrm{~h}$. After the washing step, monoclonal anti-human IgG-horseradish peroxidase (Thermo Fisher Scientific, USA) were added at an optimized dilution (1:5000) in PBS-T and incubated at $37^{\circ} \mathrm{C}$ for 1 hour. Following a final washing step, ophenylenediamine dihydrochloride substrate (Sigma Aldrich, USA) was added and the ODs were measured after $15 \mathrm{~min}$ as absorbance at $450 \mathrm{~nm}$ using an ELISA spectrophotometer (Biocrom EZ Read 400, United Kingdom). The OD readings were blanked with the PBS-T, and the cutoff value was used to discriminate between the positive and negative results. This cutoff value was based on the results of a ROC statistical analysis.

\section{Western Blotting Assay}

Lastly, a Western Blotting assay was used to confirm ELISA close to cut-off absorbance (borderline). The rTES-30 $(20 \mathrm{ug} / \mathrm{mL})$ was applied in a 12\% SDS-PAGE and electrotransferred onto a nitrocellulose membrane (GE Healthcare Life Sciences, USA) by using a transblot apparatus (Bio-Rad, USA) at $4{ }^{\circ} \mathrm{C}$ overnight. Protein transfer to membrane was checked with Ponceau S staining (Sigma-Aldrich, USA). The membrane was cut into strips and blocked with 5\% dry-milk (Nestle, Sweden) PBS-T solution for $1 \mathrm{~h}$. The strips were then incubated with serum/sera samples (diluted 1:200 in PBS-T) at $4{ }^{\circ} \mathrm{C}$ overnight, followed by monoclonal anti-human IgG-horseradish peroxidase (Thermo Fisher Scientific, USA) were added at an 
optimized dilution (1:5000) in PBS-T and incubated at $37^{\circ} \mathrm{C}$ for 1 hour. Between each step, the strips were washed with PBS-T during 5 minutes. Finally, DAB Solution (0.025\% 3,3'-Diaminobenzidine, $0.0009 \% \mathrm{H} 2 \mathrm{O} 2$ and $0.05 \mathrm{M}$ Tris $/ \mathrm{HCl}$-solution, Sigma Aldrich, USA) were used to develop the blots. Statistical analysis was performed by Pearson chi-square, two-way ANOVA and ROC curve in GraphPad software.

For positive control of each ELISA plate, we used samples seropositive to Toxocara spp. infection while also being seronegative to Ascaris lumbricoides, Trichuris trichiura, Ancylostomids, Strongyloides stercoralis, Hymenolepis nana, and Fasciola hepatica. For negative control of each ELISA plate and Western Blotting assay, we used samples seronegative to all above parasites.

\section{Ethics}

The blood banks samples studies were approved by the Research Ethics Committee in the Health Area, FURG (protocol no. 102/2012) and Research Ethics Committee of the Clinics Hospital, São Paulo University Medical School (protocol no. 0518-07).

\section{RESULTS}

Protein expression could be detected on SDS-PAGE after 72 hours of induction in methanol, while no band was detected prior to 48 hours. In addition, the intensity of the band did not change progressively post 72 hours.

After concentration technique, we could observe a unique band on SDS-PAGE (Figure 1), so an extra purification step was not needed; thus, we obtained $183.5 \mathrm{mg}$ (rTES-30) and $178.5 \mathrm{mg}$ (rTES-120) per liter of a single culture. Also, after 18 hours of deglycosylation incubation, we managed to obtain a deglycosylated product: SDS-PAGE revealed two bands, one with a higher $\mathrm{kDa}(60 \sim 70$, glycosylated) and one with lower $\mathrm{kDa}(\sim 25$, deglycosylated).

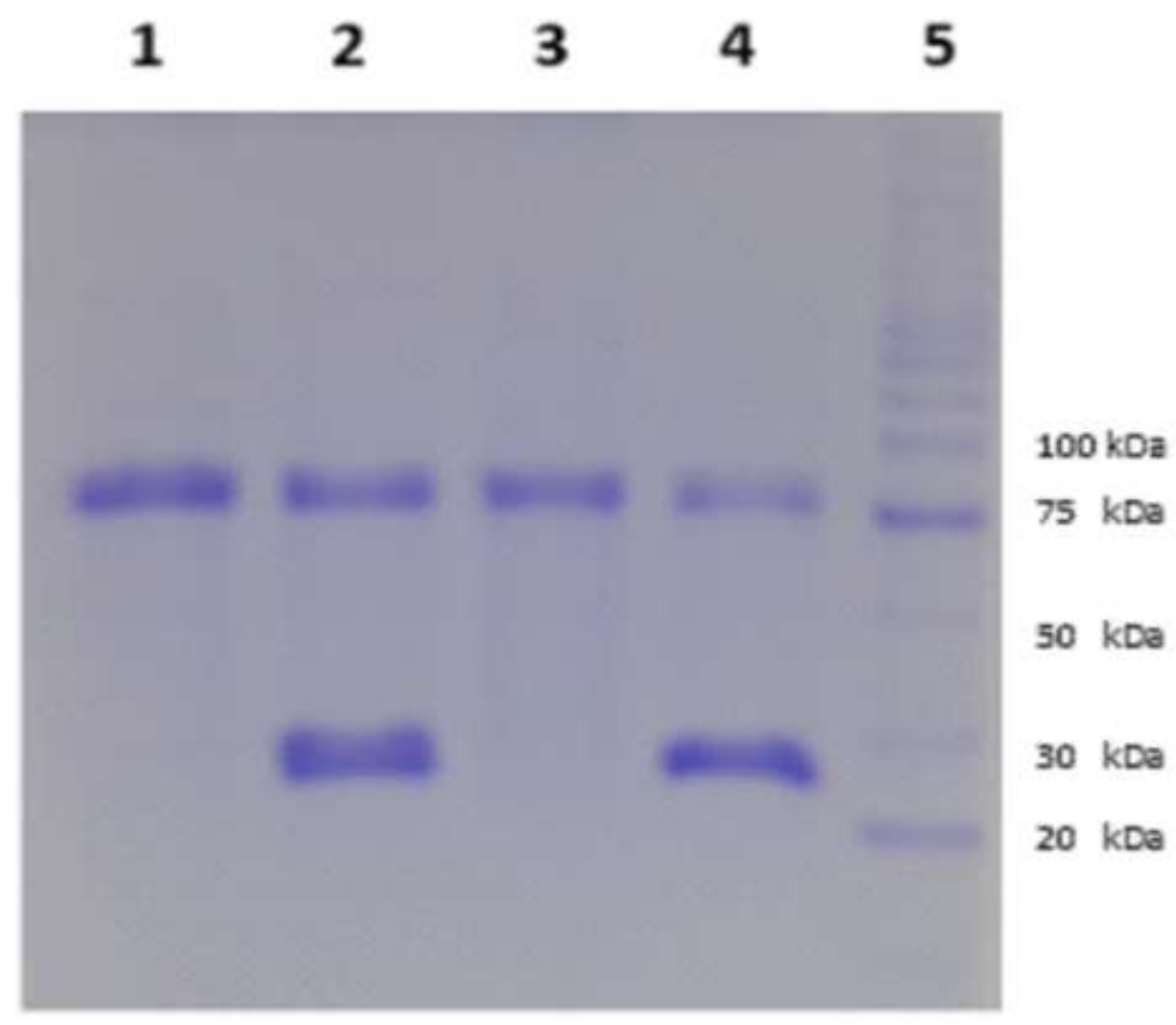

Figure 1. Evaluation of concentration and parcial deglycosylation of the recombinant proteins rTES-30 and rTES-120 on 12\% SDS-PAGE stained with Comassie Brilliant Blue R-25. Lane 1: rTES-30; 2: rTES-30 parcial deglycosylated; 3: rTES-120; 4: rTES-120 parcial deglycosylated; 5: BenchMark ${ }^{\text {TM }}$ Protein Standard (Thermo Fischer Scientific, USA).

As both rTES-30 and rTES-120 had the same apparent molecular mass in SDS-PAGE (Figure 1), we did an extra confirmation step to confirm that they were different proteins. DNA was extracted from the pellet after expression and applied on a PCR with specific primers for both genes; we observed two different bands 
that corresponded to the proteins, one with 641 base pairs (rTES-30) and one band with 506 base pairs (rTES-120).

After confirmation of the proteins, we tested their capacities to diagnose antibodies against Toxocara spp. in sera previously reported as seropositive with Toxocara spp. and seronegative to other parasites (Ascaris lumbricoides, Trichuris trichiura, Ancylostomids, Strongyloides stercoralis, Hymenolepis nana, and Fasciola hepatica), as shown in Table 1. Both proteins had the same capacity to identify positive samples $(p=0.2769)$. In addition, glycosylation did not appear to influence the diagnostic performance in rTES-30 $(p>0.9999)$ nor rTES-120 ( $p>0.9999)$.

Table 1. Optimum sensitivity and specificity for each protein after ROC analysis.

\begin{tabular}{ccc}
\hline Protein & Positive Samples (Sensitivity) & Negative Samples (Specificity) \\
\hline rTES-30 & $25 / 45(55.6 \%)$ & $43 / 45(95.6 \%)$ \\
rTES-30 Deglycosylated & $26 / 45(57.8 \%)$ & $43 / 45(95.6 \%)$ \\
rTES-120 & $31 / 45(68.9 \%)$ & $43 / 45(95.6 \%)$ \\
rTES-120 Deglycosylated & $31 / 45(68.9 \%)$ & $44 / 45(97.8 \%)$ \\
\hline
\end{tabular}

To confirm the positive results from ELISA, we performed a Western Blotting assay using Toxocara spp. positive sera. In Figure 2, we show the bands of rTES-30 and rTES-120, as well as their deglycosylated product being recognized by a patient's antibody. As a positive control, we used a previously-studied rTES120 produced in E. coli [11].

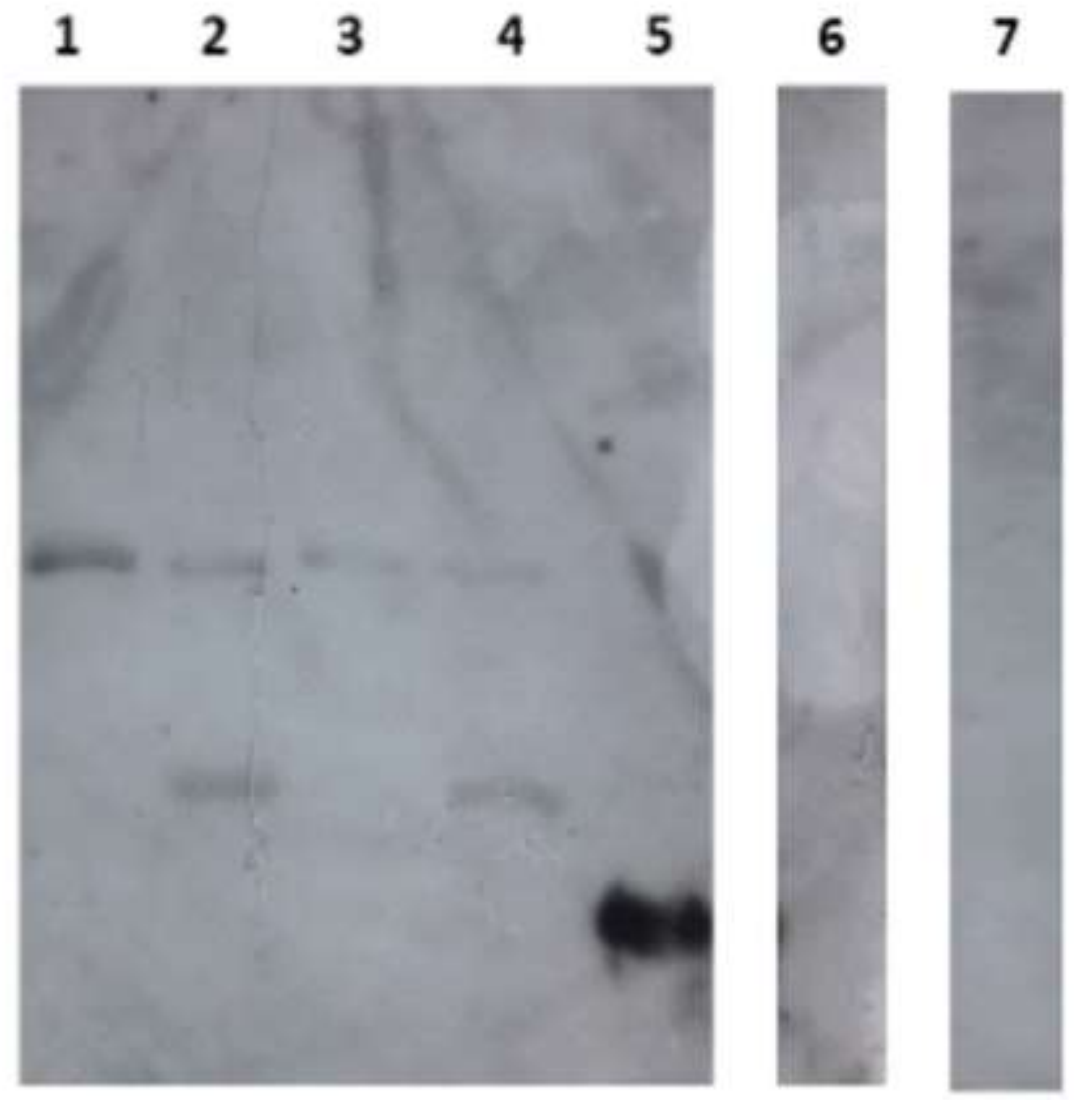

Figure 2. Performance of the recombinant antigens on a pool $(n=4)$ of positive and negative-Toxocara spp. sera on chemiluminescent Western Blotting. Lane 1: rTES-30 with positive sera; 2: rTES-30 partial deglycosylated with positive sera; 3: rTES-120 with positive sera; 4: rTES-120 partial deglycosylated with positive sera; 5: positive control (Escherichia coli recombinant TES-120, $24 \mathrm{kDa}$ ) with positive sera; 6: rTES-30 with negative sera; 7: rTES-120 with negative sera.

In Table 2, we verified the specificity of the proteins against other parasites. No difference was observed between the two proteins $(p=0.2059)$. 
Table 2. Specificity of recombinant proteins against positive anti-parasite sera with cross-reactivity potential to Toxocara spp. infection.

\begin{tabular}{|c|c|c|c|c|c|c|}
\hline 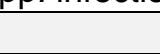 & \multicolumn{6}{|c|}{ Cross-Reactivity ${ }^{1}$} \\
\hline \multirow{3}{*}{ rTES-30 } & $\begin{array}{c}\text { Ascaris } \\
\text { lumbricoides }\end{array}$ & $\begin{array}{l}\text { Trichuris } \\
\text { trichiura }\end{array}$ & Ancylostomids & $\begin{array}{l}\text { Strongyloides } \\
\text { stercoralis }\end{array}$ & $\begin{array}{l}\text { Hymenolopis } \\
\text { nana }\end{array}$ & $\begin{array}{l}\text { Fasciola } \\
\text { hepatica }\end{array}$ \\
\hline & $0 / 9$ & $0 / 2$ & $0 / 4$ & $0 / 16$ & $0 / 6$ & $0 / 3$ \\
\hline & $0 \%$ & $0 \%$ & $0 \%$ & $0 \%$ & $0 \%$ & $0 \%$ \\
\hline \multirow[t]{2}{*}{ rTES-120 } & $3 / 9$ & $0 / 2$ & $0 / 4$ & $1 / 16$ & $0 / 6$ & $0 / 3$ \\
\hline & $33.33 \%$ & $0 \%$ & $0 \%$ & $6.25 \%$ & $0 \%$ & $0 \%$ \\
\hline
\end{tabular}

${ }^{1}$ Cut-off calculated by ROC curve assay of 40 Toxocara spp. negative patients.

\section{DISCUSSION}

In this study, we expressed rTES-30 and rTES-120 in Pichia pastoris and tested two recombinant proteins to be used in $T$. canis infection diagnostic. Prior to this study, only one other study has investigated the recombinant TES-120 produced in $P$. pastoris application to immune diagnostic of $T$. canis infection [8]. However, the study focused on the specificity of rTES-120, tested by immunoblot assay, and the topic of sensitivity was not investigated.

In the other hand, recombinant TES proteins in E. coli have been assayed previously by our group and we showed a high specificity (92.5-95\%) and sensitivity (75.6-84.4\%) [11], however E. coli expression presented a cytoplasmic protein expression that was insoluble, while $P$. pastoris have the advantage of secreting the target protein to cell exterior, being soluble $[3,6,12,13]$. As $E$. coli insoluble protein could have sensitivity issues due to denaturing requirements to solubilize it; expressing in yeast could be a valid alternative to avoid protein refolding. In this study, we present recombinant TES proteins that were successfully secreted to the medium, at an adequate level of purity, unenquiring further chromatography affinity treatment.

To verify the sensitivity and specificity of recombinant antigens produced by $P$. pastoris, we used the same tools recommended for the diagnosis of $T$. canis (ELISA and Western Blotting) [2]. While specificity was close to $100 \%$ against seropositive samples from Table 2 parasites, the sensitivity of the recombinant proteins (56\% 69\%) was not as high as proteins produced in E. coli (80 93\%), as reported by Mohamad and coauthors [3] and our previous results (75.6-84.4\%) [11]. It is important to emphasize that the same ELISA protocol and serum panel was used for both the E. coli and $P$. pastoris proteins.

Regarding specificity against other parasites, we present a product with high specificity (95.6\%), complementing the previous study by Fong and Lau [8], which evaluated specificity against cysticercosis, filariasis, malaria, amebiasis. and toxoplasmosis. In the present study, we complemented the specificity of recombinant proteins against ancylostomiasis, strongyloidiasis, hymenolepiasis, and fasciolosis; these four diseases affect populations of developing countries and, because of their cross-reactivity, they have potential to influence the diagnosis of $T$. canis infection based on native TES $[1,14,15]$.

Aside from the protein secretion mechanism, one primary difference between the two expression systems is the glycosylation capacity of $P$. pastoris. We chose to investigate whether deglycosylation could increase sensitivity $[6,12]$, but we found no statistical difference $(p>0.05)$ in sensitivity nor specificity, suggesting that the mannose products did not influence the performance of the proteins.

It is important to state that $P$. pastoris glycosylation differs from glycosylation in humans so we did not expect that the proteins produced would have the same reactivity. Specifically, $P$. pastoris provides a hypermannosylation ( $\mathrm{N}$ - and O-linked glycosylation), a simple mechanism compared to the diversity in the glycan structure assembled in the Golgi cisternae of mammals [16]. For example, Roldan and coauthors [17] reported an increase in sensitivity after deglycosylation of native TES proteins, showing that native TES does have a different glycosylation mechanism than P. pastoris.

Our main limitation to testing the deglycosylation hypothesis was that we did not manage to obtain a pure deglycosylated product, as we could still observe a glycosylated band on the SDS-PAGE (Figure 1). Nevertheless, considering that we applied a mixture of proteins in each test and that deglycosylated products were at a higher concentration (evidenced by a more intense band in SDS-PAGE, Figure 1), we should expect an increase or decrease in sensitivity, even with an "impure" product, as deglycosylated and glycosylated products should coat the microplate simultaneously [3,7]. Also, our deglycosylation methodology did not remove the O-glycosylation sites produced by Pichia pastoris, as the bands in Figure 2 were not at the same height (after deglycosylation) as the E. coli rTES-120 band and it continuous to interfere with the reaction; the presence of O-glycosylation could account for the difference between the sensitivities of $E$. coli and Pichia pastoris products, as suggested by Elefant and coauthors [18], Li and coauthors [19], and Irani and coauthors [20] studies, which collectively showed that glycosylation reduces sensitivity in serodiagnosis. 


\section{CONCLUSION}

In conclusion, the low sensitivity in the serodiagnosis diminished any advantage that $P$. pastoris expression could have. Therefore, we do not recommend $P$. pastoris recombinant TES production as an alternative for the diagnosis of toxocariasis.

Funding: This study was financed in part by Programa de Pesquisa para o SUS (PPSUS); Conselho Nacional de Desenvolvimento Científico e Tecnológico (CNPQ); Coordenação de Aperfeiçoamento de Pessoal de Nível Superior Brasil (CAPES) - Finance Code 001.

Conflicts of Interest: The authors declare no conflict of interest.

\section{REFERENCES}

1. Ma G, Holland C V, Wang T, Hofmann A, Fan CK, Maizels RM, et al. Human toxocariasis. The Lancet Infectious Diseases. 2018;18(1):e14-24.

2. Moreira GMSG, Telmo P de L, Mendonça M, Moreira ÂN, McBride AJA, Scaini CJ, et al. Human toxocariasis: Current advances in diagnostics, treatment, and interventions. Trends in Parasitology. 2014;30(9):456-64.

3. Mohamad S, Azmi NC, Noordin R. Development and evaluation of a sensitive and specific assay for diagnosis of human toxocariasis by use of three recombinant antigens (TES-26, TES-30USM, and TES-120). Journal of Clinical Microbiology. 2009;47(6):1712-7.

4. Sørensen HP, Mortensen KK. Soluble expression of recombinant proteins in the cytoplasm of Escherichia coli. Microb Cell Fact. 2005;4:1.

5. Jia B, Jeon $\mathrm{CO}$. High-throughput recombinant protein expression in Escherichia coli : current status and future perspectives. Open Biology. 2016;6(8):160196.

6. Macauley-Patrick S, Fazenda ML, McNeil B, Harvey LM. Heterologous protein production using the Pichia pastoris expression system. Yeast. 2005;22(4):249-70.

7. Długosz E, Wiśniewski M. Toxocara canis glycans influence antigen recognition by mouse $\lg \mathrm{G} 1$ and $\operatorname{lgM}$ antibodies. Acta Parasitologica. 2016;61(1).

8. Fong M-Y, Lau Y-L. Recombinant expression of the larval excretory-secretory antigen TES-120 of Toxocara canis in the methylotrophic yeast Pichia pastoris. Parasitology Research. 2004;92(2):173-6.

9. Sambrook J, Green MR. Molecular Cloning - A laboratory Manual. 4º ed. New York: Cold Spring Harbor; 2012. $2231 \mathrm{pp}$.

10. Dummer LA, Araujo IL, Campos FS, Rosa MC da, Finger PF, Oliveira PD de, et al. Development of an Indirect ELISA for Serological Diagnosis of Bovine herpesvirus 5. PLOS ONE. 2016;11(2):e0149134.

11. Santos LMD, Magalhães CG, Telmo PL, Cerqueira MP, Donassolo RA, Leite FPL, et al. Sensitivity and specificity of recombinant proteins in Toxocara spp. for serodiagnosis in humans: Differences in adult and child populations. PLOS ONE. 2018;13(12): e0208991.

12. Balamurugan V, Reddy GR, Suryanarayana VVS. Pichia pastoris: A notable heterologous expression system for the production of foreign proteins - Vaccines. Indian Journal of Biotechnology. 2007;6(2):175-86.

13. Cantu-Bustos JE, Vargas-Cortez T, Morones-Ramirez JR, Balderas-Renteria I, Galbraith DW, McEvoy MM, et al. Expression and purification of recombinant proteins in Escherichia coli tagged with the metal-binding protein CusF. Protein Expression and Purification. 2016;121:61-5.

14. Momčilović $S$, Cantacessi $C$, Arsić-Arsenijević $V$, Otranto $D$, Tasić-Otašević $S$. Rapid diagnosis of parasitic diseases: current scenario and future needs. Clinical Microbiology and Infection. 2018;

15. Lu X-T, Gu Q-Y, Limpanont Y, Song L-G, Wu Z-D, Okanurak K, et al. Snail-borne parasitic diseases: an update on global epidemiological distribution, transmission interruption and control methods. Infectious Diseases of Poverty. 2018;7(1):28.

16. Ahmad M, Hirz M, Pichler H, Schwab H. Protein expression in Pichia pastoris: recent achievements and perspectives for heterologous protein production. Appl Microbiol Biotechnol. 2014;98(12):5301-17.

17. Roldán WH, Elefant GR, Ferreira AW. Deglycosylation of Toxocara excretory-secretory antigens improves the specificity of the serodiagnosis for human toxocariasis. Parasite Immunology. 2015;37(11):557-67.

18. Elefant GR, Roldán WH, Seeböck A, Kosma P. Evaluation of a di-O-methylated glycan as a potential antigenic target for the serodiagnosis of human toxocariasis. Parasite Immunology. 2016;38(4):236-43.

19. Li D, Schaewen M von, Wang X, Tao W, Zhang Y, Li L, et al. Altered Glycosylation Patterns Increase Immunogenicity of a Subunit Hepatitis C Virus Vaccine, Inducing Neutralizing Antibodies Which Confer Protection in Mice. Journal of Virology. 2016;90(23):10486-98.

20. Irani ZA, Kerkhoven EJ, Shojaosadati SA, Nielsen J. Genome-scale metabolic model of Pichia pastoris with native and humanized glycosylation of recombinant proteins. Biotechnol Bioeng. 2016;113(5):961-9. 